\title{
Motion Correction Strategies for Integrated PET/MR
}

\author{
Sebastian Fürst ${ }^{1,2}$, Robert Grimm ${ }^{3,4}$, Inki Hong ${ }^{5}$, Michael Souvatzoglou ${ }^{1}$, Michael E. Casey ${ }^{5}$, Markus Schwaiger ${ }^{1}$, \\ Stephan G. Nekolla*1, and Sibylle I. Ziegler*1 \\ ${ }^{1}$ Department of Nuclear Medicine, Technische Universität München, Munich, Germany; ${ }^{2}$ Graduate School of Information Science in \\ Health (GSISH), Technische Universität München, Munich, Germany; ${ }^{3}$ Pattern Recognition Lab, Friedrich-Alexander-Universität \\ Erlangen-Nürnberg, Erlangen, Germany; ${ }^{4}$ Siemens Healthcare MR, Erlangen, Germany; and ${ }^{5}$ Siemens Healthcare MI, Knoxville, \\ Tennessee
}

Integrated whole-body PET/MR facilitates the implementation of a broad variety of respiratory motion correction strategies, taking advantage of the strengths of both modalities. The goal of this study was the quantitative evaluation with clinical data of different MR- and PET-data-based motion correction strategies for integrated PET/MR. Methods: The PET and MR data of 20 patients were simultaneously acquired for 10 min on an integrated PET/MR system after administration of ${ }^{18} \mathrm{~F}-\mathrm{FDG}$ or ${ }^{68} \mathrm{Ga}$-DOTANOC. Respiratory traces recorded with a bellows were compared against MR self-gating signals and signals extracted from PET raw data with the sensitivity method, by applying principal component analysis (PCA) or Laplacian eigenmaps and by using a novel variation combining the former and either of the latter two. Gated sinograms and MR images were generated accordingly, followed by image registration to derive MR motion models. Corrected PET images were reconstructed by incorporating this information into the reconstruction. An optical flow algorithm was applied for PETbased motion correction. Gating and motion correction were evaluated by quantitative analysis of apparent tracer uptake, lesion volume, displacement, contrast, and signal-to-noise ratio. Results: The correlation between bellows- and MR-based signals was $0.63 \pm 0.19$, and that between MR and the sensitivity method was $0.52 \pm 0.26$. Depending on the PET raw-data compression, the average correlation between MR and PCA ranged from $0.25 \pm 0.30$ to $0.58 \pm 0.33$, and the range was $0.25 \pm 0.30$ to $0.42 \pm 0.34$ if Laplacian eigenmaps were applied. By combining the sensitivity method and PCA or Laplacian eigenmaps, the maximum average correlation to MR could be increased to $0.74 \pm 0.21$ and $0.70 \pm 0.19$, respectively. The selection of the best PET-based signal for each patient yielded an average correlation of $0.80 \pm 0.13$ with MR. Using the best PET-based respiratory signal for gating, mean tracer uptake increased by $17 \pm 19 \%$ for gating, $13 \pm 10 \%$ for MRbased motion correction, and $18 \pm 15 \%$ for PET-based motion correction, compared with the static images. Lesion volumes were $76 \pm 31 \%$, $83 \pm 18 \%$, and $74 \pm 22 \%$ of the sizes in the static images for gating, MR-based motion correction, and PET-based motion correction, respectively. Conclusion: Respiratory traces extracted from MR and PET data are comparable to those based on external sensors. The proposed PET-driven gating method improved respiratory signals and overall stability. Consistent results from MR- and PET-based correction methods enable more flexible PET/MR scan protocols while achieving higher PET image quality.

Received Aug. 8, 2014; revision accepted Dec. 10, 2014

For correspondence or reprints contact: Sebastian Fürst, Department of Nuclear Medicine, Technische Universität München, Ismaninger Strasse 22, 81675 München, Germany.

E-mail: sebastian.fuerst@tum.de

${ }^{*}$ Contributed equally to this work.

Published online Jan. 8, 2015.

COPYRIGHT (c) 2015 by the Society of Nuclear Medicine and Molecular Imaging, Inc.
Key Words: PET/MR; multimodal imaging; respiratory gating; motion correction

J Nucl Med 2015; 56:261-269

DOI: 10.2967/jnumed.114.146787

$\mathbf{P}$ hysiologic motion is a major source of deterioration of image quality in PET, leading to image blurring and rendering tumor uptake quantification less accurate and lesion volume delineation more difficult (1). Various methods for the reduction of motion artifacts in PET images have been proposed, including gating (1), image-based approaches (2-4), and the incorporation of motion information into the reconstruction algorithm $(5,6)$. Gating constitutes a tradeoff of scan duration and image quality, because only a fraction of the acquired PET coincidences is considered for the reconstruction of individual gates. This leads to lower sensitivity, worse statistics, and higher noise. Prolongation of examinations improves image quality but adversely affects patient comfort and throughput. Despite this disadvantage, gating is still the most widely used respiratory motion compensation scheme.

For the correction of respiratory motion in PET, two types of information are essential, vector fields describing the motion within the body of the patient and a respiratory signal, which establishes the connection between motion model and PET data. The integration of whole-body PET with MR imaging and its introduction into the clinical routine facilitates the implementation of a broad variety of motion-correction strategies, taking advantage of the respective strengths of both modalities. The respiratory signal can be obtained with external sensors, such as respiratory belts, or cameras in combination with fiducial markers mounted on the chest of the patient $(1,7,8)$ or can be extracted from PET data (9-11). In MR, navigator echoes are used to track the position and phase shifts of objects of interest in the field of view (FOV) that are subject to respiratory motion, mostly the liver dome (12). With regard to the acquisition of motion vector fields, MR-based motion modeling techniques (13) appear to be the logical choice because of their analysis of physical deformation of anatomic structures for the detection of motion. However, other methods were published in recent years that promise to derive the flow of activity directly from PET data (14). MR-based PET motion correction is widely assumed to be more robust because of the anatomic information and higher signal-to-noise ratio (SNR) of MR images but is otherwise invasive and costly. Already-demanding PET/MR workflows and scan protocols (15) 
would have to be extended for motion-modeling scans, and the acquisition of respiratory signals would block the subsystem with dedicated MR sequences throughout the entire PET examination. Alternatively, all clinical MR sequences would have to be interleaved with navigators, which might not be possible for any sequence with the sampling frequency required for respiratory gating or without inducing image artifacts. These factors may put further constraints on PET/MR protocols, limiting patient comfort and throughput. In contrast, PET-driven motion correction and its implementation may appear straightforward. However, it is still considered less accurate and reliable because of the higher noise of PET data, subject to counting rate statistics and tracer kinetics. None of the published studies of motion correction included a direct comparison of the two types of motion correction. The respective authors derived their results either from animal studies (16) or with simulated or phantom data (17-22). PET-driven motion correction was assessed with the data of 14 patients (14), whereas MR-based motion correction has so far been evaluated with the data of 5 patients at most $(23,24)$.

Therefore, the goal of this study was the evaluation of different motion-correction strategies for integrated PET/MR. Within the scope of the study, we propose a variation of methods for the extraction of respiratory signals from PET list-mode data, testing the results on the clinical data of 20 patients against MR- and sensorbased gating methods. Additionally, a rigorous comparative study of MR- and PET-driven motion-corrected PET image reconstruction was performed in terms of tracer uptake quantification, lesion volume definition, and image quality with the clinical data of 14 patients.

\section{MATERIALS AND METHODS}

\section{Motion Correction Strategies}

Respiratory Gating. As PET and possibly MR data have to be gated retrospectively, exact knowledge of the corresponding respiratory state is required at any time point during the examination. Within the scope of this study, this was achieved by the acquisition of respiratory signals according to 5 methods, that is, the pressure-sensitive respiratory bellows (resp_bellows) that is shipped with the scanner by default, a prototype implementation of a self-gated MR imaging pulse sequence (resp_mr) $(25,26)$, the PET-based sensitivity method (resp_sens) $(9,10)$, and the application of dimensionality reduction techniques such as principal component analysis (PCA) or Laplacian eigenmaps to the sinogram space (resp_pca and resp_le, respectively) $(11,27,28)$. Before execution of the PET-based methods, the list-mode streams were divided into nonoverlapping 200-ms time frames. The processing steps of resp_pca and resp_le are outlined in Figure 1. The number of sinogram planes was reduced to 127 by single-slice rebinning. All but the central 176 radial bins of each projection were cropped because of low counts and marginal respiratory motion. Seven levels of

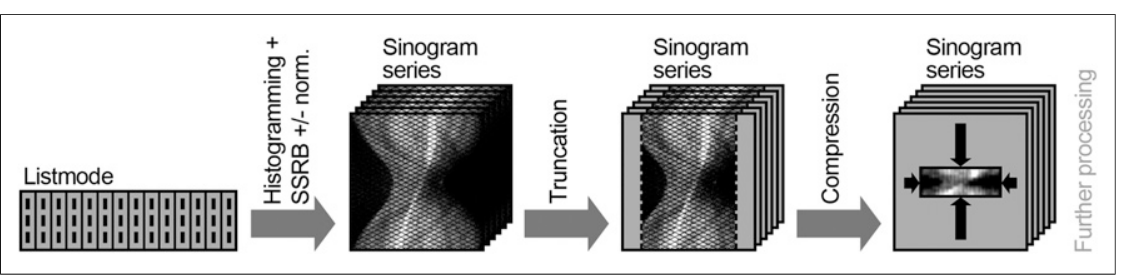

FIGURE 1. Schematic of workflow for extraction of respiratory signals from PET data using dimensionality reduction techniques. radial compression and angular mashing were evaluated, ranging from 44 bins/9 projections to $1 \mathrm{bin} / 1$ projection. PCA and Laplacian eigenmaps were applied to the sinogram series using the MATLAB Toolbox for Dimensionality Reduction (29). We proposed and developed a fifth method, which builds on resp_pca/resp_le but integrates resp_sens (resp_pca + sens, resp_le + sens) by omitting the normalization in single-slice rebinning of sinogram bin values to the number of lines of response contributing to the corresponding plane. Thus, the axial sensitivity profile of the PET detector system was preserved in sinogram space. Apart from the normalization, processing for resp_pca+sens and resp_le+sens was identical to that for resp_pca and resp_le, respectively.

Calculation of Motion Vectors. Sets of motion vector fields $M$ describing the intergate displacement of morphologic structures are required for the warping of image volumes and acquired volumes of attenuation correction factors from the reference to the other respiratory states as defined by the gates. For MR-based motion correction (moco_mr), $M$ is calculated by acquisition of MR images at each gate and subsequent nonrigid registration. In the present study, the inverse motion vector fields $M^{-1}$ from gate to reference were generated by estimating for each voxel in the target image volume the corresponding voxel index in the reference after backward transformation to the reference. Special care was taken that spatial mismatch between an original voxel in the reference image and the corresponding voxel after the full warp cycle (from reference to gate to reference) was minimal, since such inconsistency would lead to a loss of resolution and adverse effects on image quantification in motion-corrected iterative PET image reconstruction.

In the absence of other imaging modalities, motion information can be derived from PET images using optical flow algorithms (14). The PET-driven method in this study (moco_pet) is based on a massconserving algorithm with an improved constraint, which assumes equal overall activity in each image (14).

Motion-Corrected Iterative PET Reconstruction. It is desirable to incorporate the two types of previously described information into the process of image reconstruction instead of manipulating reconstructed images. The most straightforward approach extends the conventional iterative ordered-subset expectation maximization algorithm by integrating a warp function into the forward and backward projectors (Fig. 2).

If the most suitable methods for each of these steps were to be combined, a motion correction strategy could be optimized for specific clinical requirements.

\section{Patient Population}

The respiratory gating and motion correction methods were applied to 20 patients (11 women and 9 men) who had been referred to our department for the diagnosis and staging of malignant diseases (11 cases in the abdomen, 1 in the heart, and 8 in the thorax) using ${ }^{18} \mathrm{~F}$-FDG PET/ CT or ${ }^{18} \mathrm{~F}$-FDG PET/MR (18 patients) or using ${ }^{68} \mathrm{Ga}$-DOTANOC PET/ MR (2 patients). The patients were $64 \pm 14 \mathrm{y}$ old (mean \pm SD) and weighed $76 \pm 15 \mathrm{~kg}$. The diseases include breast, liver, and pancreatic cancer, with additional lesions in, for example, lungs, lymph nodes, esophagus, and small intestine.

All patients gave written informed consent to participate in this study and, if only a PET/CT examination was clinically required and scheduled, to undergo a second scan using PET/MR. The approval of the institutional review board (project 2967/10) and the radiation protection authorities had been obtained. No additional radiotracers were injected after the first scan. 


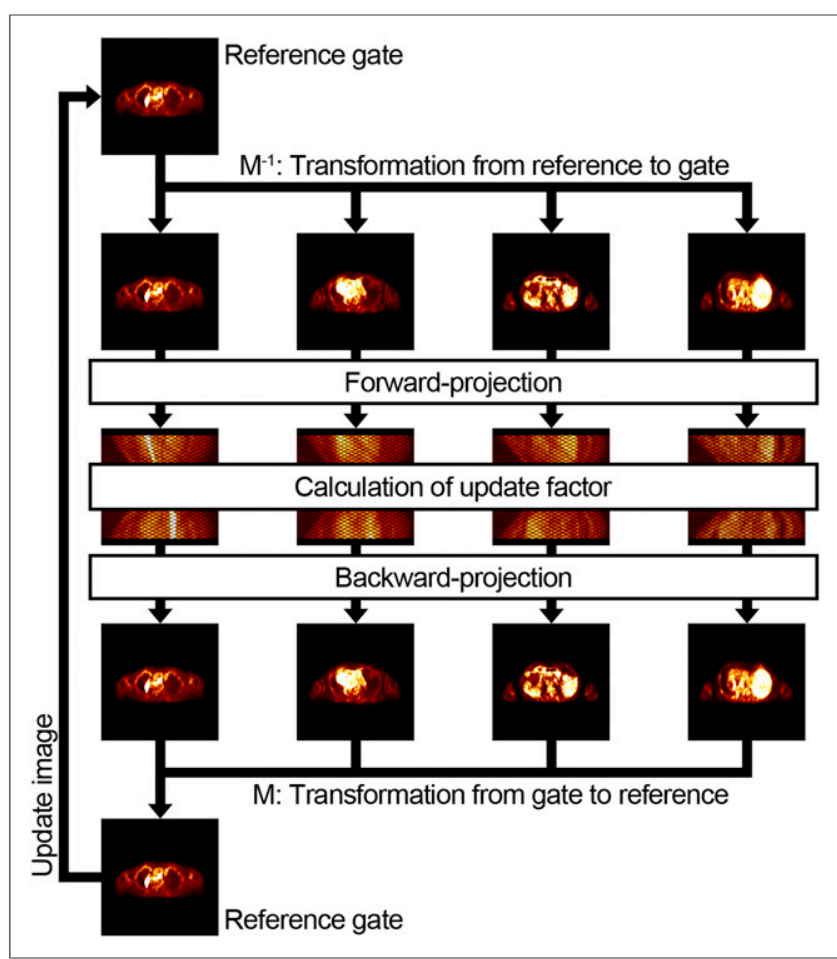

FIGURE 2. Schematic of workflow for incorporation of motion information into ordered-subset expectation maximization reconstruction algorithm.

\section{Acquisition}

Instrumentation. All data in this study were acquired on a 3-T avalanche-photodiode-based integrated clinical PET/MR system (Biograph mMR; Siemens Healthcare; software version VB18P). The PET component of this tomograph is located between the gradient and the body coils. It covers axial and transaxial FOVs of 25.8 and $59.4 \mathrm{~cm}$, respectively. Each detector block contains a matrix of $8 \times 8$ lutetium oxyorthosilicate crystals with a size of $4 \times 4 \times 20 \mathrm{~mm}$, which are read out by $3 \times 3$ avalanche photodiodes. PET spatial resolution was measured to be $4.3 \mathrm{~mm}$ at a radial distance of $1 \mathrm{~cm}$ from the FOV center, and the PET sensitivity was $15.0 \mathrm{kcps} / \mathrm{MBq}$ in the FOV center $(30,31)$. Apart from the standard body coil and spine array coils, a flexible 6element surface coil (Body Matrix Coil; Siemens Healthcare) was used for the MR measurements.

Imaging Protocol. Patients were injected with $339 \pm 63 \mathrm{MBq}$ of ${ }^{18} \mathrm{~F}$ FDG and scanned $136 \pm 24$ min after injection, or they were injected with 93 or $122 \mathrm{MBq}$ of ${ }^{68} \mathrm{Ga}$-DOTANOC and scanned 66 or $52 \mathrm{~min}$, respectively, after injection. Before a scan, the patient was positioned on the scanner bed with the arms beside the torso and the respiratory bellows attached between the costal arch and sternum to improve the signal for abdominal or thoracic breathing. One or two of the body array coils were placed on the abdomen or thorax, depending on the size of the patient and the body region to be examined. This region was then centered in the FOV of the scanner. A prototypical T1-weighted radial stack-of-stars spoiled 3-dimensional gradient echo sequence with fat suppression (sagittal slab orientation; FOV, $400 \times 400 \times 360 \mathrm{~mm}$; spatial resolution, $1.65 \times 1.65 \times 5 \mathrm{~mm}$; matrix, 256 pixels; 72 slices; $61 \%$ slice resolution; $5 / 8$ partial Fourier) was used for the subsequent derivation and calculation of MR-based respiratory signals and motion vector fields. After generation of an attenuation map ( $\mu$ map) with a 2-point Dixon 3-dimensional volumetric interpolated breath-hold T1-weighted MR imaging sequence (repetition time/first echo time, $3.6 \mathrm{~ms} / 1.23 \mathrm{~ms}$; FOV, $500 \times 399 \mathrm{~mm}$; voxel size, $4.1 \times 2.6 \times 3.1 \mathrm{~mm}$; 128 slices; flip angle, $10.0^{\circ}$; acquisition time, $19 \mathrm{~s}$ ), for which patients were given commands to hold their breath at end-expiration, PET list-mode and radial MR data were simultaneously acquired for $10 \mathrm{~min}$.

\section{Processing}

Respiratory Signals and PET Gating. All recorded respiratory traces were resampled to $10 \mathrm{~Hz}$ and then normalized for correlation analysis according to the following equation, where $y(t)$ is the signal height at a time point $t$ and $\mu(Y)$ and $\sigma(Y)$ the average height and corresponding SD of the entire respiratory trace $Y$ :

$$
y_{\text {norm }}(t)=\frac{y(t)-\mu(Y)}{\sigma(Y)} \text {. }
$$

List-mode events were binned into sets of gated sinograms by means of variable-amplitude-based gating (32) according to the produced respiratory signals. The number of gates was set to 5 , which constituted a good compromise between captured respiratory motion and noise in the reconstructed PET images and has been identified as appropriate in the literature (33). Sinograms comprising PET data from the entire scan (static) were also generated for each patient.

Motion-Field Estimation. Radial MR readouts were partitioned according to resp_mr, following the steps previously outlined for PET gating, and corresponding gated MR images were reconstructed. A nonrigid registration algorithm proposed for lung imaging (34) was applied for the calculation of motion vectors. The gate comprising data recorded at end-expiration was selected as the reference, since it is least affected by intragate motion under the current gating scheme.

Attenuation Correction. Patient $\mu$ maps were created by segmenting the fat and water images generated by the Dixon MR imaging sequence. The original $\mu$ maps with 4 tissue classes (35) were then used for the reconstruction of images that formed the basis of the respiratory gating analysis. For the reconstruction of PET images at end-inspiration, motion vector fields were applied to deform the $\mu$ maps to this state. For the evaluation of motion correction, all voxels in the $\mu$ maps classified as fat were assigned the attenuation coefficient of soft tissue, and morphologic structures in the lungs at or near the location of lesions were removed. This measure was taken to isolate the outcome of motion correction from adverse effects caused by mismatch of attenuation and emission data at tissue boundaries. Truncation of arms in $\mu$ maps because of the limited FOV of the MR subsystem was compensated for by maximum-likelihood reconstruction of attenuation and activity (36). For the reconstruction of attenuation-corrected PET images at end-inspiration, motion vector fields were additionally applied to deform the $\mu$ maps from the reference to this state.

PET Image Reconstruction. Static, gated (reference state, gate 1, endexpiration; gate 5, end-inspiration) and motion-corrected (reference state, end-expiration) PET images were reconstructed from all previously described sinograms or sets of sinograms using the corresponding original and modified $\mu$ maps and following the clinical standard in our department (3-dimensional ordered-subset expectation maximization, 3 iterations, 21 subsets, $172 \times 172$ matrix, 4.0-mm gaussian postreconstruction filter) (31). In addition to attenuation correction, data were also normalized and corrected for dead time, scatter, decay, frame length, and randoms.

\section{Analysis}

The respiratory signals of all 20 study patients were evaluated. Of these 20 patients, only those with moving lesions that exhibited tracer uptake sufficient for unambiguous segmentation in all static, gated, and motion-corrected images were included for subsequent image-based analysis of respiratory gating and motion correction, resulting in 14 patient datasets with a total of 27 lesions. For the validation of respiratory gating, only one lesion was considered per patient to avoid bias. 
Respiratory Signals. The processed respiratory traces were evaluated qualitatively and by calculating the Pearson correlation coefficients of resp_mr with all other methods.

Image Quantification. The effects of gating and motion correction on reconstructed images were analyzed in terms of apparent tracer uptake concentration in suspected tumor lesions and background tissue. For this purpose, isocontour volumes of interest (VOI) were segmented for each lesion individually by using a region-growing algorithm (isocontour threshold, 50\%). Apart from the maximum activity concentration, the average activity concentration $A$ in each isocontour VOI was calculated, as well as $\mathrm{SD} \sigma$, contrast $C$, and SNR. The latter were defined in a way similar to that of a previous study (37):

$$
\begin{gathered}
C=\frac{A_{\text {lesion }}-A_{\text {background }}}{A_{\text {background }}} . \\
\mathrm{SNR}=\frac{A_{\text {lesion }}-A_{\text {background }}}{\sigma_{\text {background }}} .
\end{gathered}
$$

For the analysis of background tissue, one rectangular cuboidal VOI per patient was defined in the liver under the constraint that the VOI could not include edge voxels or focal tracer uptake. The thus maximum possible size was additionally restricted to $11 \times 11 \times 21$ voxels $(4.6 \times 4.6 \times 4.3 \mathrm{~cm})$. Image noise was then expressed as the relative SD of all voxel values in the background VOI.

Lesion Displacement and Volumes. The position of an isocontour VOI along the craniocaudal axis was computed as its center of mass as previously described (38). Lesion displacement $\Delta z$ was measured for the evaluation of respiratory gating as the absolute difference in positions between gates 1 and 5 . Lesion volume $V$ was defined as the total volume of all voxels within the isocontour VOI in the reference gate.

Statistical Analysis. The 2-sided paired Wilcoxon signed-rank test was performed to determine the statistical significance of the results. Differences for which $P$ values of 0.05 or more were calculated were regarded as statistically insignificant.

\section{RESULTS}

\section{Respiratory Signals}

For all 20 patients, respiratory traces could be successfully generated (Fig. 3). The positions of inhalation peaks and the overall noise of resp_bellows and resp_mr were visually comparable and consistent. However, because of the limited recorded range of signal heights as currently implemented on the PET/MR scanner, inhalation peaks in resp_bellows were frequently cut off. Therefore, resp_mr was chosen as the reference signal.

The average coefficients of correlation were $0.63 \pm 0.19$ between resp_bellows and resp_mr (maximum, 0.94; minimum, 0.13 ) and $0.52 \pm 0.26$ between resp_sens and resp_mr (maximum, 0.86; minimum, 0.01). Dimensionality reduction techniques yielded average correlations between $0.25 \pm 0.30$ and $0.42 \pm 0.34$ for resp_mr/resp_le and between $0.25 \pm 0.30$ and $0.58 \pm 0.33$ for resp_mr/resp_pca, depending on the level of mashing and radial compression. The preservation of the sensitivity profile in the processed data increased the maximum average correlation of resp_le+sens to resp_mr to $0.70 \pm 0.19$. The application of PCA to a sinogram space with 44 radial bins and 3 projections with the sensitivity profile preserved, which will be denoted as resp_pca443sens in the remainder of the paper, resulted in the highest average correlation, $0.74 \pm 0.21$ (maximum, 0.93; minimum, 0.06), between resp_mr and any PETdriven extraction method. If the PET-based extraction method

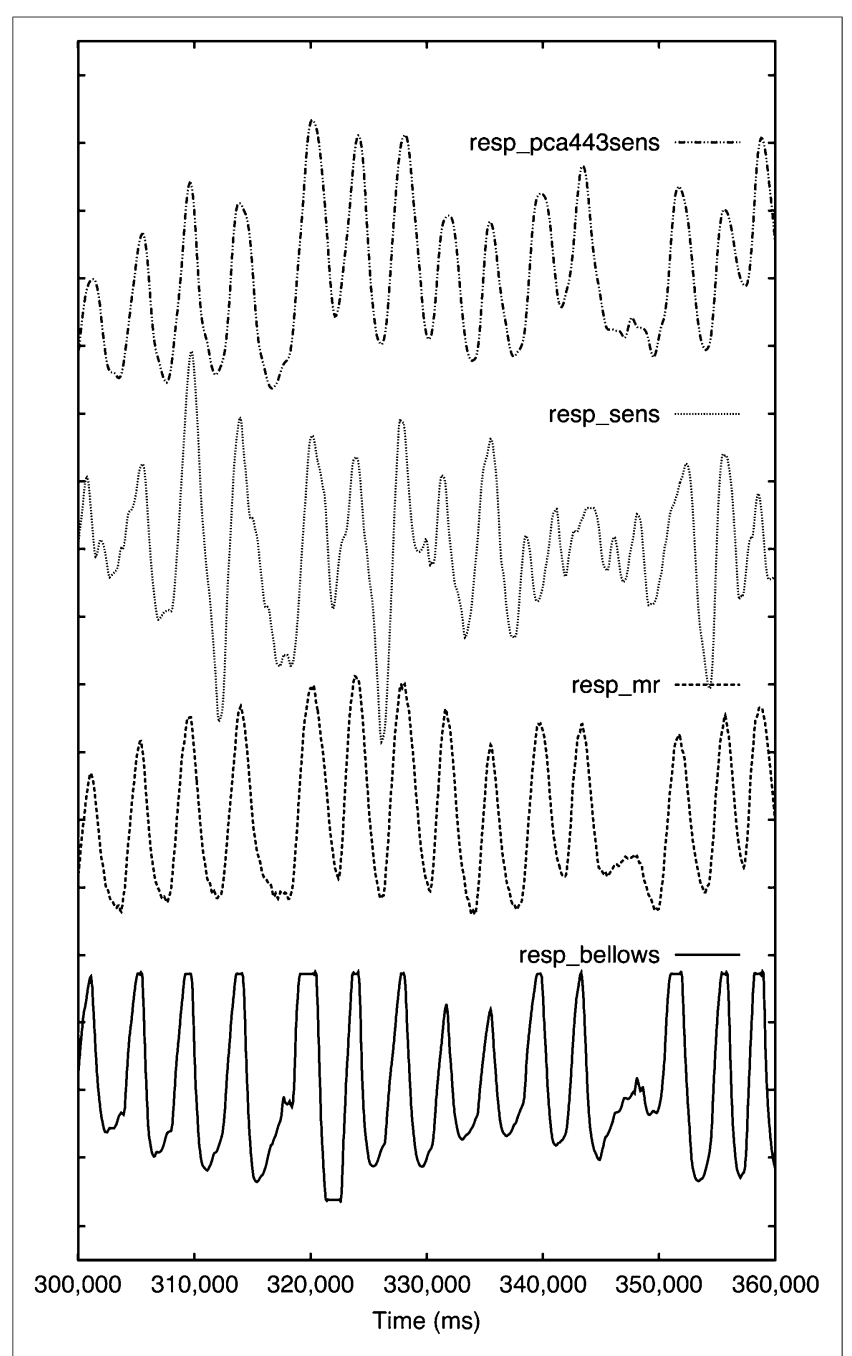

FIGURE 3. Respiratory traces of patient 8 from 60 -s mid-scan window. Correlation coefficients with resp_mr as reference were 0.71 (resp_bellows), 0.79 (resp_sens), and 0.92 (resp_pca443sens). Resp_ bellows is cut off above a certain signal height, whereas resp_sens appears noisiest. Visual impression confirms high correlation between resp_mr and resp_pca443sens.

that presented the highest correlation to resp_mr was selected for each patient individually, which will be referred to as resp_bestpet in the remainder of the paper, an even higher correlation of $0.80 \pm$ 0.13 (maximum, 0.93; minimum, 0.40) was achieved on average for the entire patient population. The coefficient of correlation to resp_mr was higher than 0.8 for $15 \%, 15 \%, 55 \%$, and $65 \%$ of all 20 patients for resp_bellows, resp_sens, resp_pca443sens, and resp_bestpet, respectively (Fig. 4). Complete information on all levels of compression is in Table 1.

On the basis of these results, gated and motion-corrected images were reconstructed from gated sinograms created according to resp_bellows, resp_mr, resp_sens, and resp_bestpet. Corresponding coefficients of correlation to resp_mr for the group of patients included in the image-based analysis were $0.71 \pm 0.11$ for resp_bellows, $0.55 \pm$ 0.20 for resp_sens, and $0.81 \pm 0.09$ for resp_bestpet. Images based on resp_pca443sens (correlation to resp_mr, $0.80 \pm 0.10$ ) were not evaluated separately because of the similarly high quality of resp_pca443sens and resp_bestpet for the included patients. 


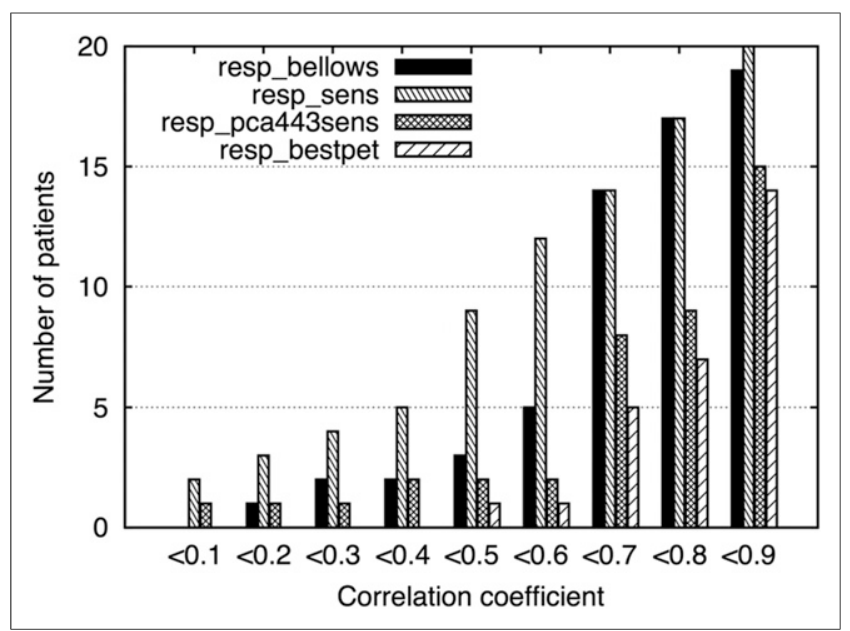

FIGURE 4. Cumulative frequency histogram of coefficients of correlation of resp_bellows, resp_sens, resp_pca443sens, and resp_bestpet, with resp_mr as reference. Correlation coefficient is higher than 0.6 for $75 \%$ (resp_bellows), 40\% (resp_sens), 90\% (resp_pca443sens), and 95\% (resp_bestpet) of all 20 patients.

The quantitative analysis of gated images is summarized in Table 2, and an example is shown in Figure 5.

\section{Motion-Corrected Iterative PET Reconstruction}

Both methods of motion correction included in this study improved the visual impression of the reconstructed PET images (Fig. 6). The size and location of morphologic structures in gated images and both of the motion-corrected images were consistent. In static images, the same structures appeared blurred and larger. However, image noise was significantly lower in moco_mr and moco_ pet than in gated images, whereas the noise patterns in static and motion-corrected images were virtually indistinguishable. Complete quantitative results on motion correction can be found in Table 3 .

Static images exhibited a background noise level of $14.7 \pm$ $2.5 \%$ on average. Depending on the respiratory-signal source, this level could be maintained at between $14.3 \pm 2.4 \%$ and $14.5 \pm$ $2.6 \%$ for moco_mr and at between $14.8 \pm 2.3 \%$ and $15.0 \pm 2.4 \%$ for moco_pet in motion-corrected images but was between $29.2 \pm$ $4.2 \%$ and $29.9 \pm 4.5 \%$ in the gated images.
For resp_bestpet, none of the observed differences in tracer uptake between gated and moco_mr images (maximum uptake, $P=0.140$; mean uptake, $P=0.274$ ), between gated and moco_pet images (maximum uptake, $P=0.990$; mean uptake, $P=0.572$ ), or between moco_mr and moco_pet images (maximum uptake, $P=0.116$; mean uptake, $P=0.153$ ) were significant.

The differences in lesion volumes (gating method, resp_bestpet) were not significant for gated and moco_mr images $(P=0.058)$ or for gated and moco_pet images $(P=0.909)$. For moco_mr and moco_pet, a $P$ value of 0.029 was calculated.

In terms of contrast and SNR, moco_mr and moco_pet were not significantly different $(P \geq 0.167)$ with resp_bestpet as the gating method, whereas the application of any motion correction improved both figures significantly over gating $(P \leq 0.028)$.

\section{DISCUSSION}

The goal of this study was the assessment of different MR- and PET-data-driven respiratory gating and motion correction methods.

With regard to respiratory signals, the quality of resp_bellows was artificially reduced by the limited signal range supported in the current implementation on the PET/MR scanner and the resulting signal cutoff. Although this factor should not affect amplitude-based gating in most cases, the true correlation of resp_mr and resp_bellows is expected to be higher than the $0.63 \pm 0.19$ found in this study.

The comparably low average correlation of resp_sens with resp_mr, $0.52 \pm 0.26$, combined with a higher SD across the patient population reveals its lower reliability. Büther et al. (10) reported a correlation of 0.65 between their implementation of resp_sens and respiratory signals obtained with a video-based method. Whereas the injected activity doses were similar, patients included in the present study had more than $30 \%$ lower activity levels at the start of the scan because the average delay was $135 \mathrm{~min}$ instead of $60 \mathrm{~min}$, which might explain the difference in correlation. Moreover, Büther et al. restricted their study to cardiac scans, for which an extended hot object, that is, the heart, is centered in the FOV, which could be an additional advantage for resp_sens. Support for this possibility can be found in a publication by Thielemans et al. (28), who observed a correlation of approximately 0.50 between resp_sens and a video-based method if one third of the acquired counts was deliberately rejected for the simulation of lower doses.

TABLE 1

Dependence of PET-Based Respiratory Trace Quality on Sinogram-Space Compression

\begin{tabular}{cccccc}
\hline & & \multicolumn{3}{c}{ Correlation coefficients $^{*}$} \\
\cline { 3 - 6 } Radial bins & Projections & resp_le & resp_pca & resp_le+sens & resp_pca+sens \\
\hline 1 & 1 & $0.25 \pm 0.30$ & $0.25 \pm 0.30$ & $0.51 \pm 0.29$ & $0.52 \pm 0.29$ \\
\hline 1 & 1 & $0.30 \pm 0.32$ & $0.33 \pm 0.34$ & $0.70 \pm 0.19$ & $0.73 \pm 0.14$ \\
22 & 1 & $0.34 \pm 0.34$ & $0.40 \pm 0.34$ & $0.63 \pm 0.25$ & $0.71 \pm 0.17$ \\
44 & 1 & $0.31 \pm 0.32$ & $0.36 \pm 0.34$ & $0.56 \pm 0.30$ & $0.67 \pm 0.24$ \\
44 & 3 & $0.42 \pm 0.34$ & $0.56 \pm 0.34$ & $0.60 \pm 0.24$ & $0.74 \pm 0.21$ \\
44 & 6 & $0.39 \pm 0.32$ & $0.58 \pm 0.33$ & $0.54 \pm 0.25$ & $0.71 \pm 0.25$ \\
& 9 & $0.37 \pm 0.30$ & $0.56 \pm 0.34$ & $0.46 \pm 0.29$ & $0.69 \pm 0.26$
\end{tabular}

*Reference: resp_mr.

Shown values are averaged over entire patient population (mean \pm SD). 
TABLE 2

Complete Results from Quantitative Image-Based Analysis of Respiratory Gating Methods

\begin{tabular}{|c|c|c|c|c|c|}
\hline Parameter & Static & Resp_bellows & Resp_mr & Resp_sens & Resp_bestpet \\
\hline \multicolumn{6}{|c|}{ Maximum activity concentration } \\
\hline Static (kBq/mL) & $21.7 \pm 17.5$ & - & - & - & - \\
\hline Gate 1 (\% of static) & - & $113.5 \pm 11.6$ & $116.9 \pm 15.5$ & $110.4 \pm 9.1$ & $117.1 \pm 15.6$ \\
\hline \multicolumn{6}{|c|}{ Mean activity concentration } \\
\hline Static $(\mathrm{kBq} / \mathrm{mL})$ & $14.4 \pm 11.6$ & - & - & - & - \\
\hline Gate 1 (\% of static) & - & $111.3 \pm 10.1$ & $114.8 \pm 13.8$ & $109.8 \pm 7.9$ & $114.3 \pm 11.4$ \\
\hline \multicolumn{6}{|l|}{ Volume } \\
\hline Static $\left(10^{3} \mathrm{~mm}^{3}\right)$ & $6.5 \pm 8.9$ & - & - & - & - \\
\hline Gate 1 (\% of static) & - & $80.1 \pm 23.5$ & $76.6 \pm 24.6$ & $81.3 \pm 19.2$ & $73.5 \pm 30.3$ \\
\hline \multicolumn{6}{|l|}{ Displacement } \\
\hline Gate 1 - gate $5(\mathrm{~mm})$ & - & $4.4 \pm 2.6$ & $5.2 \pm 3.0$ & $3.8 \pm 3.3$ & $5.4 \pm 2.6$ \\
\hline
\end{tabular}

Of the more complex PET methods evaluated in this paper, resp_pca yielded better results than resp_le. Our hypothesis could be confirmed that preservation of the sensitivity profile followed by the application of PCA or Laplacian eigenmaps as proposed above increased the quality and robustness of PET-driven respiratory signal extraction, especially toward lower activities in the patient's body or with less specific tracer uptake. In general, the higher the quality of resp_le/resp_pca was, the lower was the benefit of resp_le+sens/resp_pca+sens. However, integration of the sensitivity method facilitated higher levels of compression without sacrificing the resulting signal quality, which means that information lost because of compression could be compensated for. This leads to the possibility of extracting respiratory signals from PET list-mode data under more difficult conditions, for example, lower injected doses, and reduction of processing time or required computing power.

As shown with resp_bestpet, the signal quality could be increased with patient-specific compression parameters. This observation hints at a sensitivity of data-driven respiratory signal extraction to a combination of breathing patterns, activity levels, and tracer distributions, which are very individual. Hence, there is no single level of sinogram compression that yields the best result for every patient examination. The comparably low minimum correlation coefficient of 0.4 for resp_bestpet to resp_mr could be treated as an outlier. If this data point is excluded, the lowest

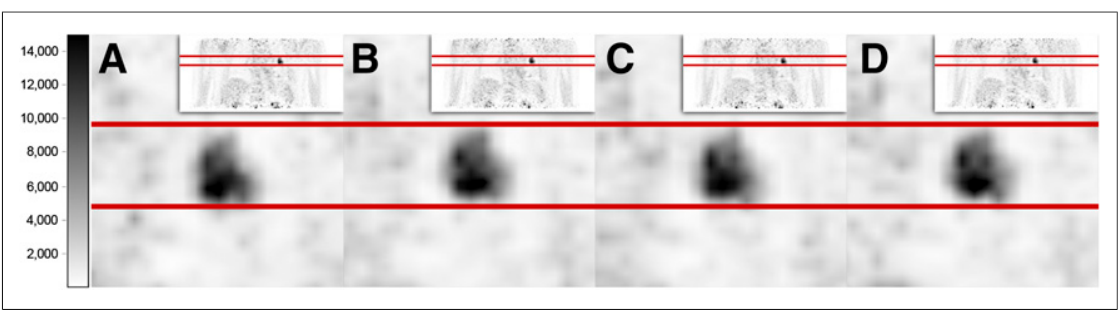

FIGURE 5. Coronal slices through lesion of patient 8, in images (gate 1) gated according to resp_bellows (A), resp_mr (B), resp_sens (C), and resp_pca443sens (D). Visual differences in lesion volume and location among $B, C$, and $D$ are negligible. Compared with other images, lesion appears to be slightly shifted in A, possibly indicating higher degree of intragate motion. correlation will be 0.67 . Furthermore, $75 \%$ of resp_bestpet signals provide correlation coefficients higher than 0.70 , and in $30 \%$ of the cases they are even higher than 0.90 . Consequently, resp_bestpet was confirmed to be a feasible approach, if both MR and PET data are available for a common period.

The correlation with the video-based method of approximately 0.8 for both resp_pca and resp_le as reported by Thielemans et al. $(11,28)$ could be achieved only with resp_le+sens/resp_pca + sens in the present study. Possible reasons for this discrepancy include the levels of sinogram compression that were used and the sampling rates of the respiratory signals, the latter of which were 5 times higher in this study. Moreover, because the studies were not performed on the same scanner models, the corresponding results might be subject to different scanners' sensitivities and scatter fractions. The longer scan duration of $10 \mathrm{~min}$ in the present study, compared with the $3 \mathrm{~min}$ of Thielemans et al., might also play a role.

Quantitative image analysis confirmed the correlation analysis. The only significant differences in lesion displacement were between resp_bellows and resp_pca443sens and between resp_sens and the other gating methods under investigation, and the only significant differences in lesion volume were between resp_bellows and resp_pca443sens or resp_bestpet. These findings support the notion that resp_bellows and resp_sens produce slightly inferior respiratory signals.

The results of motion correction had to be validated with both static and gated images. Gated images represent the reference for tracer uptake quantification and lesion volume. This should be achieved with motion correction, albeit at the noise levels of static images. As expected, both motion correction methods fully recovered the static noise level, whereas the noise exhibited by gated images was significantly higher, reducing SNR and hence lesion detectability.

The inferiority of motion correction driven by PET to that driven by MR 


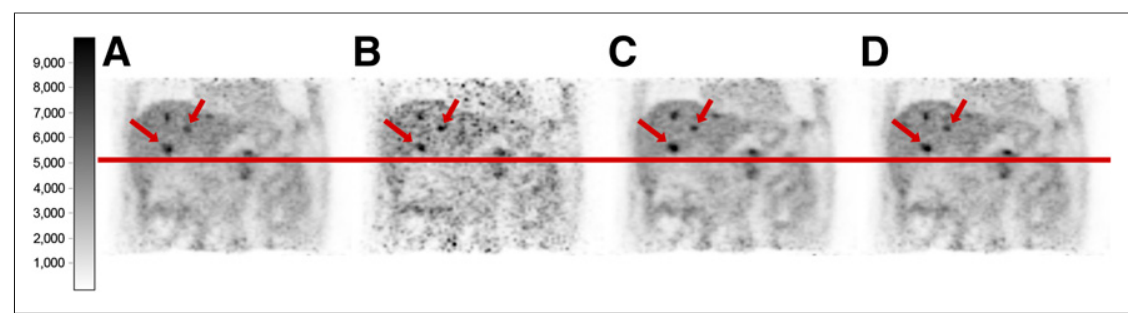

FIGURE 6. Coronal slices through static (A), gated (gate 1) (B), moco_mr (C), and moco_pet (D) images of patient 4. Gating was performed in all cases according to resp_pca446sens. Motion blurring of lesions (liver, arrows) was significantly reduced by gating and motion correction, leading to smaller volumes and higher apparent uptake. Reduced number of counts is obvious in $B$ and noise patterns in $A, C$, and $D$ are consistent.

could not be confirmed in this study. On the contrary, the only statistically significant difference between the two motion correction methods for any of the analyzed image properties was found in lesion volumes, if sinograms were created using resp_bestpet. Moreover, moco_pet appears to perform marginally better than moco_mr in terms of tumor uptake and volume. This could be due to two reasons. First, the applica- tion of MR-derived motion fields to PET data constitutes an indirect approach, whereas optical flow allows the direct calculation of motion information from PET data. The result of the latter would be a better agreement between the motion-corrected images and the images of the reference gate. Subsequently, the quality of the motion-corrected image would increase with the image quality of the gated reference. Second, MR motion fields were created by image warping using trilinear interpolation. Otherwise, it would be difficult to connect information from multiple modalities. However, mass-preserved optical flow, which forms the basis of moco_ pet, is superior to optical flow using linear interpolation as shown by Dawood et al. (14).

As supposed, SNR increased significantly over gating after the application of either motion correction method. The case was similar for lesion contrast, for which only moco_pet images combined with resp_pca443sens did not change significantly. In

TABLE 3

Summary of Results from Image-Based Analysis of Motion Correction Methods

\begin{tabular}{|c|c|c|c|c|c|}
\hline Parameter & Static & Resp_bellows & Resp_mr & Resp_sens & Resp_bestpet \\
\hline \multicolumn{6}{|c|}{ Maximum activity concentration } \\
\hline Static $(\mathrm{kBq} / \mathrm{mL})$ & $18.6 \pm 11.9$ & - & - & - & - \\
\hline Gate 1 (\% of static) & - & $119.9 \pm 16.8$ & $119.3 \pm 15.5$ & $114.7 \pm 15.0$ & $116.4 \pm 18.3$ \\
\hline Moco_mr (\% of static) & - & $109.2 \pm 9.4$ & $112.2 \pm 10.2$ & $104.1 \pm 5.9$ & $111.1 \pm 8.3$ \\
\hline Moco_pet (\% of static) & - & $110.4 \pm 15.6$ & $114.1 \pm 16.8$ & $110.7 \pm 15.6$ & $116.8 \pm 16.5$ \\
\hline \multicolumn{6}{|l|}{ Mean activity concentration } \\
\hline Static (kBq/mL) & $12.5 \pm 8.0$ & - & - & - & - \\
\hline Gate 1 (\% of static) & - & $120.5 \pm 17.8$ & $119.6 \pm 15.5$ & $115.5 \pm 17.0$ & $116.5 \pm 19.0$ \\
\hline Moco_mr (\% of static) & - & $110.5 \pm 10.3$ & $113.6 \pm 11.9$ & $104.7 \pm 6.3$ & $112.7 \pm 9.8$ \\
\hline Moco_pet (\% of static) & - & $110.3 \pm 15.6$ & $115.3 \pm 14.7$ & $111.2 \pm 13.7$ & $117.7 \pm 15.4$ \\
\hline \multicolumn{6}{|l|}{ Volume } \\
\hline Static $\left(10^{3} \mathrm{~mm}^{3}\right)$ & $4.0 \pm 6.5$ & - & - & - & - \\
\hline Gate 1 (\% of static) & - & $69.5 \pm 25.9$ & $70.3 \pm 25.1$ & $74.1 \pm 23.1$ & $75.8 \pm 30.6$ \\
\hline Moco_mr (\% of static) & - & $83.7 \pm 18.4$ & $82.2 \pm 18.5$ & $91.7 \pm 16.4$ & $82.7 \pm 18.4$ \\
\hline Moco_pet (\% of static) & - & $84.7 \pm 21.1$ & $77.3 \pm 19.5$ & $82.6 \pm 20.1$ & $74.0 \pm 21.7$ \\
\hline \multicolumn{6}{|l|}{ Contrast } \\
\hline Static (\%) & $47.3 \pm 31.2$ & - & - & - & - \\
\hline Gate 1 (\% of static) & - & $108.0 \pm 92.2$ & $97.6 \pm 100.5$ & $109.1 \pm 53.6$ & $113.3 \pm 59.4$ \\
\hline Moco_mr (\% of static) & - & $106.4 \pm 43.7$ & $107.5 \pm 51.6$ & $116.3 \pm 52.6$ & $110.2 \pm 42.7$ \\
\hline Moco_pet (\% of static) & - & $112.1 \pm 31.9$ & $112.1 \pm 18.9$ & $104.0 \pm 9.2$ & $114.8 \pm 25.0$ \\
\hline \multicolumn{6}{|l|}{ SNR } \\
\hline Static (\%) & $1,300 \pm 1,396$ & - & - & - & - \\
\hline Gate 1 (\% of static) & - & $65.9 \pm 61.7$ & $60.3 \pm 66.4$ & $65.7 \pm 41.0$ & $71.3 \pm 40.8$ \\
\hline Moco_mr (\% of static) & - & $119.3 \pm 60.8$ & $125.6 \pm 73.8$ & $120.9 \pm 52.3$ & $126.7 \pm 62.6$ \\
\hline Moco_pet (\% of static) & - & $119.6 \pm 35.2$ & $125.9 \pm 30.6$ & $114.3 \pm 21.1$ & $132.8 \pm 40.1$ \\
\hline
\end{tabular}

Data are mean \pm SD. 
general, these results highlight the consistency of both MR- and PET-driven motion correction methods.

Because of the limited number of real patient cases included in published studies, comparison is difficult. Würslin et al. evaluated an MR-based motion correction of reconstructed PET images with data of 5 patients (23). Similar to our findings, they observed increases of $28 \%, 25 \%$, and $27 \%$ in motion-corrected relative to static images for maximum activity concentration in lesions, contrast, and SNR, respectively. They defined contrast as the ratio of maximum activity in a lesion-not mean activity (our definition)— over mean activity in background tissue. Because of the corresponding behavior of mean and maximum tracer uptake in our study, this difference should not play an important role, however. When comparing motion-corrected and gated images, Würslin et al. found only SNR to improve significantly, whereas maximum tracer uptake, volume, and contrast were significantly worse. This finding contrasts with our observation and may hint at the advantages of motion correction incorporated into the reconstruction algorithm, where in the present study for both evaluated methods contrast and SNR were significantly better than in gated images but tracer uptake and volume were not significantly different (resp_bestpet). Petibon et al. (24) reconstructed motioncorrected images of one liver case using MR-derived motion information. They reported comparable results, that is, an increase in target-to-background ratio, which was defined identically to contrast as used by Würslin, of between $22 \%$ and $45 \%$, whereas apparent lesion volumes decreased by between $13 \%$ and $29 \%$, depending on the lesion, if compared with static images. The results for PET-driven motion correction as published by Dawood et al. (14) relate to myocardial thickness and blood-pool activity in the left ventricle in cardiac cases only and are therefore not comparable.

The consistency of MR- and PET-based motion correction methods as established in our study offers the operator of a PET/ MR system a range of strategy choices tailored to a specific application. In oncologic ${ }^{18}$ F-FDG studies, there will be the highest flexibility with regard to motion correction and scan protocols as the user can fully rely on PET-driven gating and correction methods. In more specialized cases with lower applied activities or less common radiotracers, moco_pet might have its limitations. For such purposes, motion models could be acquired with, for example, radial MR sequences. During this span of a few minutes, MR- and PET-derived respiratory signals could be compared, and depending on the correlation, it could be decided if PET respiratory signal quality is sufficient. If not, the system could automatically fall back to resp_mr or resp_bellows or a different external sensor. If in another scenario the user is not concerned with scan time, because the planned MR protocol is not demanding or because the clinician would be satisfied with the anatomic quality of the MR images acquired with the self-gating pulse sequence for motion modeling, moco_mr could be utilized in combination with resp_mr.

In future work, the limitations of PET-driven gating and moco_pet with regard to counting rate statistics should be thoroughly investigated. Moreover, the comparison of moco_mr and moco_pet should be extended to other more specialized applications, such as cardiac PET imaging. In addition, it would be desirable to develop an algorithm to automatically select the optimal combination of radial bins and projections for resp_pca, as well as subsequent quality assessment without additional MR information.

\section{CONCLUSION}

Respiratory traces extracted from PET data are comparable to MR-derived signals and those based on external sensors. With the proposed PET-driven gating method, a higher quality of respiratory traces was achieved and the overall stability improved. Improvements in image quality, tracer uptake quantification, and lesion volume delineation achieved with MR- and PET-based respiratory motion correction methods were consistent in evaluated oncologic standard examinations, allowing for more flexible PET/MR scan protocols that use solely PET-driven monitoring and correction. In lower-dose regimes, the results of motion correction could be enhanced by the addition of external sensors or motion models derived from MR sequences.

\section{DISCLOSURE}

The costs of publication of this article were defrayed in part by the payment of page charges. Therefore, and solely to indicate this fact, this article is hereby marked "advertisement" in accordance with 18 USC section 1734. The PET/MR system used for this study was funded through Deutsche Forschungsgemeinschaft (DFG) Grossgeräteinitiative 2010. This research was also supported by the Graduate School of Information Science in Health (GSISH) and the TUM Graduate School, as well as by the Sonderforschungsbereich (SFB) 824. The research leading to these results has received funding from the European Union Seventh Framework Program (FP7) under 294582 ERC grant MUMI. No other potential conflict of interest relevant to this article was reported.

\section{ACKNOWLEDGMENTS}

We thank Claudia Meisinger, Sylvia Schachoff, and Anna Winter for their valuable support and assistance with patient preparation and examination, as well as Jorge Cabello, Isabel Dregely, and Matthias Fenchel for discussions.

\section{REFERENCES}

1. Nehmeh SA, Erdi YE, Ling CC, et al. Effect of respiratory gating on quantifying PET images of lung cancer. $J$ Nucl Med. 2002;43:876-881.

2. Bundschuh RA, Martínez-Möller A, Essler M, Nekolla SG, Ziegler SI, Schwaiger M. Local motion correction for lung tumours in PET/CT: first results. Eur J Nucl Med Mol Imaging. 2008;35:1981-1988.

3. Fulton RR, Meikle SR, Eberl S, Pfeiffer J, Constable CJ, Fulham MJ. Correction for head movements in positron emission tomography using an optical motiontracking system. IEEE Trans Nucl Sci. 2002;49:116-123.

4. Picard Y, Thompson CJ. Motion correction of PET images using multiple acquisition frames. IEEE Trans Med Imaging. 1997;16:137-144.

5. Rahmim A, Cheng JC, Dinelle K, et al. System matrix modeling of externally tracked motion. Proc IEEE Nucl Sci Symp Med Imaging Conf. 2006;4:21372141 .

6. Lamare F, Carbayo MJL, Cresson T, et al. List-mode-based reconstruction for respiratory motion correction in PET using non-rigid body transformations. Phys Med Biol. 2007;52:5187-5204.

7. Wagman R, Yorke E, Ford E, et al. Respiratory gating for liver tumors: use in dose escalation. Int J Radiat Oncol. 2003;55:659-668.

8. Li XA, Stepaniak C, Gore E. Technical and dosimetric aspects of respiratory gating using a pressure-sensor motion monitoring system. Med Phys. 2006;33: $145-154$.

9. He J, O'Keefe GJ, Gong SJ, et al. A novel method for respiratory motion gated with geometric sensitivity of the scanner in 3D PET. IEEE Trans Nucl Sci. 2008;55:2557-2565. 
10. Büther F, Dawood M, Stegger L, et al. List mode-driven cardiac and respiratory gating in PET. J Nucl Med. 2009;50:674-681.

11. Thielemans K, Rathore S, Engbrant F, Razifar P. Device-less gating for PET/ CT using PCA. Proc IEEE Nucl Sci Symp Med Imaging Conf. 2011;39043910.

12. Ehman RL, Felmlee JP. Adaptive technique for high-definition MR imaging of moving structures. Radiology. 1989;173:255-263.

13. McClelland JR, Hawkes DJ, Schaeffter T, King AP. Respiratory motion models: a review. Med Image Anal. 2013;17:19-42.

14. Dawood M, Gigengack F, Jiang X, Schäfers KP. A mass conservation-based optical flow method for cardiac motion correction in 3D-PET. Med Phys. 2013;40:012505.

15. Martinez-Möller A, Eiber M, Nekolla SG, et al. Workflow and scan protocol considerations for integrated whole-body PET/MRI in oncology. J Nucl Med. 2012;53:1415-1426.

16. Chun SY, Reese TG, Ouyang J, et al. MRI-based nonrigid motion correction in simultaneous PET/MRI. J Nucl Med. 2012;53:1284-1291.

17. Guérin B, Cho S, Chun SY, et al. Nonrigid PET motion compensation in the lower abdomen using simultaneous tagged-MRI and PET imaging. Med Phys. 2011;38:3025-3038.

18. Polycarpou I, Tsoumpas C, Marsden PK. Analysis and comparison of two methods for motion correction in PET imaging. Med Phys. 2012;39:6474-6483.

19. Polycarpou I, Tsoumpas C, King AP, Marsden PK. Impact of respiratory motion correction and spatial resolution on lesion detection in PET: a simulation study based on real MR dynamic data. Phys Med Biol. 2014;59:697-713.

20. Dikaios N, Izquierdo-Garcia D, Graves MJ, Mani V, Fayad ZA, Fryer TD. MRIbased motion correction of thoracic PET: initial comparison of acquisition protocols and correction strategies suitable for simultaneous PET/MRI systems. Eur Radiol. 2012;22:439-446.

21. Dikaios N, Fryer TD. Registration-weighted motion correction for PET. Med Phys. 2012;39:1253-1264.

22. Petibon Y, Fakhri El G, Nezafat R, Johnson N, Brady T, Ouyang J. Towards coronary plaque imaging using simultaneous PET-MR: a simulation study. Phys Med Biol. 2014;59:1203-1222.

23. Würslin C, Schmidt H, Martirosian P, et al. Respiratory motion correction in oncologic PET using T1-weighted MR imaging on a simultaneous whole-body PET/MR system. J Nucl Med. 2013;54:464-471.

24. Petibon Y, Huang C, Ouyang J, et al. Relative role of motion and PSF compensation in whole-body oncologic PET-MR imaging. Med Phys. 2014;41:042503.

25. Chandarana H, Block TK, Rosenkrantz AB, et al. Free-breathing radial 3D fatsuppressed T1-weighted gradient echo sequence. Invest Radiol. 2011;46:648653.
26. Grimm R, Fürst S, Souvatzoglou M, et al. Self-gated MRI motion modeling for respiratory motion compensation in integrated PET/MRI. Med Image Anal. 2014;19:110-120.

27. Razifar P, Axelsson J, Schneider H, Langstrom B, Bengtsson E, Bergstrom M. Volume-wise application of principal component analysis on masked dynamic PET data in sinogram domain. IEEE Trans Nucl Sci. 2006;53:2759-2768.

28. Thielemans K, Schleyer PJ, Marsden PK, Manjeshwar RM, Wollenweber SD, Ganin A. Comparison of different methods for data-driven respiratory gating of PET data. Proc IEEE Nucl Sci Symp Med Imaging Conf. 2013;1-4.

29. van der Maaten L, Postma EO, van den Herik HJ. Dimensionality reduction: a comparative review. Tilburg University website. http://www.tilburguniversity. edu/upload/59afb3b8-21a5-4c78-8eb3-6510597382db_TR2009005.pdf. Accessed December 15, 2014.

30. Delso G, Fürst S, Jakoby BW, et al. Performance measurements of the Siemens mMR integrated whole-body PET/MR scanner. J Nucl Med. 2011;52:19141922.

31. Drzezga A, Souvatzoglou M, Eiber M, et al. First clinical experience with integrated whole-body PET/MR: comparison to PET/CT in patients with oncologic diagnoses. J Nucl Med. 2012;53:845-855.

32. Dawood M, Buther F, Lang N, Schober O, Schäfers KP. Respiratory gating in positron emission tomography: a quantitative comparison of different gating schemes. Med Phys. 2007;34:3067-3076.

33. Park S-J, Ionascu D, Killoran J, et al. Evaluation of the combined effects of target size, respiratory motion and background activity on 3D and 4D PET/CT images. Phys Med Biol. 2008;53:3661-3679.

34. Heinrich MP, Jenkinson M, Brady SM, Schnabel JA. Globally optimal deformable registration on a minimum spanning tree using dense displacement sampling. In: Ayache N, Delingette H, Golland P, Mori K, eds. Medical Image Computing and Computer-Assisted Intervention-MICCAI 2012. New York, NY: Springer; 2012:115-122.

35. Martinez-Möller A, Souvatzoglou M, Delso G, et al. Tissue classification as a potential approach for attenuation correction in whole-body PET/MRI: evaluation with PET/CT data. J Nucl Med. 2009;50:520-526.

36. Nuyts J, Dupont P, Stroobants S, Benninck R, Mortelmans L, Suetens P. Simultaneous maximum a posteriori reconstruction of attenuation and activity distributions from emission sinograms. IEEE Trans Med Imaging. 1999;18:393-403.

37. Kinahan PE, Karp JS. Figures of merit for comparing reconstruction algorithms with a volume-imaging PET scanner. Phys Med Biol. 1994;39:631-642.

38. Bundschuh RA, Martínez-Möller A, Essler M, et al. Postacquisition detection of tumor motion in the lung and upper abdomen using list-mode PET data: a feasibility study. J Nucl Med. 2007;48:758-763. 